\title{
Clinical Evaluation of Efficacy and Safety of a Herbal Formulation in Benign Prostatic Hyperplasia: A Single Blind, Randomized, Placebo-Controlled Study ${ }^{*}$
}

\author{
Ramanathan Jeyaraman ${ }^{1}$, Pralhad S. Patki ${ }^{2 \#}$ \\ ${ }^{1}$ Department of Urology, Madras Medical College, Chennai, India \\ ${ }^{2}$ The Himalaya Drug Company, Bangalore, India \\ Email: "dr.patki@himalayahealthcare.com
}

Received July 4, 2012; revised August 6, 2012; accepted August 26, 2012

\begin{abstract}
Benign prostatic hyperplasia (BPH) is a condition intimately related to ageing. Currently available treatment options for the management of BPH have various limitations and associated adverse effects. A polyherbal formulation is claimed to be beneficial in patients with benign prostatic hyperplasia. This single blind, placebo-controlled study evaluated the clinical efficacy and safety of polyherbal formulation in BPH. Material and Methods: A total of 60 patients who were diagnosed as BPH and who were willing to give informed consent were included in the study. At the randomization visit, a detailed medical history was obtained and the patients underwent a thorough systemic examination and digital rectal examination. Routine blood analysis, urinalysis and serum levels of prostate specific antigen were carried out. Abdominal pelvic ultrasonography was done at entry and after completing the study. The severity of the urinary parameters was evaluated using American Urological Association symptom score. All the patients were randomized using random table into either polyherbal group $(n=30)$ or placebo $(n=30)$. Each patient received either polyherbal formulation or placebo in a dose of 2 capsules twice a day with meals for two months. All adverse events reported by the patients or observed by investigators were recorded. Statistical analysis was done according to the intention-to-treat principles. Analysis was performed between the groups using Fisher's exact test or unpaired " $\mathrm{t}$ " test (Independent $\mathrm{t}$-test). Results: Fifty-six patients completed the study. There was a significant improvement in the mean AUA symptom score, PVR urine volume urinary hesitancy, intermittent flow, straining during urination, sense of incomplete micturition and frequency of night-time urination, in the polyherbal formulation group. Four patients from the placebo group withdrew from the study due to lack of benefit to the treatment. Conclusion: The beneficial clinical efficacy of polyherbal formulation observed in this study in the management of BPH could be due to the synergistic actions of its potent herbs. This polyherbal formulation was well tolerated and safe.
\end{abstract}

Keywords: Phytotherapy; Ayurveda; Clinical Evaluation

\section{Introduction}

The prostate is about the size of a walnut in men younger than 30 years [1], situated at the base of the bladder, deep in the male pelvis, and surrounds the proximal portion of the urethra. Prostate size increases gradually leading to benign prostatic hyperplasia (BPH) in most men older than 60 years [2]. Benign prostatic hyperplasia is a nonmalignant enlargement of the prostate that is due to excessive cellular growth of both the glandular and the stromal elements of the gland in the periurethral zone of the prostate [3]. BPH is nearly universal result of the aging process in men.

*Financial support: The Himalaya Drug Company, Makali, Bangalore562123 (India).

${ }^{\#}$ Corresponding author.
The principal hypothesis for the hypertrophic reaction of prostate tissue is steroid mediated cellular proliferation and inflammatory response to local infection. Also inefficiency of apoptotic machinery and aberrant stromalepithelial interactions are also suggested to probably contribute to the abnormal growth of prostate [4].

As men age, the level of circulating testosterone decreases, while the number of androgen receptors increases, causing the overgrowth of the prostate [5]. The result is an enlarged prostate gland that causes bladder outflow obstruction. Because the enlarged gland obstructs the prostatic urethra, the patient strains during urination to overcome the obstruction. Over time, straining to urinate causes the detrusor muscle of the bladder to thicken and diverticula to form in the bladder. When the detrusor muscle can no longer generate sufficient 
pressure to overcome the urethral obstruction, the bladder fails and retains urine. Destruction of striated and smooth muscles results in urinary incontinence [6].

Symptoms of BPH can be either irritative or obstructive. Obstructive symptoms include straining, hesitancy, weak stream, intermittency, and sense of incomplete bladder emptying and irritative symptoms include urgency, frequency, and nocturia [7].

Based on the American Urological Association (AUA) symptom score prostate can be classified as mild, moderate and severe. Symptoms are measured on a scale of 1 to 35. A score of 1 to 7 is classified as mild symptoms, 8 to 19 signify moderate symptoms, and a score from 20 to 35 signifies severe symptoms [8].

The AUA recommends routine urinalysis for the diagnosis of BPH. Urinalysis can reveal pyuria and bacteriuria suggesting infection, hematuria suggesting inflammation or urothelial malignancy, or active urinary sediment suggesting postobstructive nephropathy. Optional studies include serum creatinine and prostate specific antigen (PSA) [9]. The PSA is a strong predictor of prostate volume in BPH [10] and level increases at advanced stage of the disease and helpful in monitoring at later stage for clinical management [11].

Although most of the patients seek medical care for the symptoms of $\mathrm{BPH}$, urinary tract obstruction due to longstanding BPH can cause some major health problems such as bleeding from the prostate, recurrent infections, bladder stones, inability to urinate, and renal insufficiency.

The goal of therapy is to reduce or alleviate lower urinary tract symptoms, to prevent complications, and to minimize the adverse effects of treatment [12]. Watchful waiting is the preferred management strategy for patients with mild symptoms and also an appropriate option for men with moderate to severe symptoms who have not yet developed complications of BPH.

Currently available drugs for treatment of BPH are $\alpha 1$-adrenergic antagonists (tamsulosin, alfuzosin, doxazosin and terazosin) and $5 \alpha$-reductase inhibitors (finasteride and dutasteride). These medications act on dynamic and static components of bladder outlet obstruction [13,14]. $\alpha 1$-Adrenergic antagonists act on the dynamic component of bladder outlet obstruction are the initial choice of medical treatment in most men with BPH. In men with large prostate glands ( $>40 \mathrm{~mL}$ ), higher PSA levels, and moderate to severe symptoms, treatment with $5 \alpha$-reductase inhibitors or combination therapy is considered [15].

Open surgical removal of prostatic adenoma remained the gold standard treatment of BPH for many decades. The conversion from open to transurethral surgery occurred gradually [16]. Minimally invasive procedures are performed in more advanced degrees of outlet obstruc- tion. In patients in whom medical and minimally invasive options for BPH have been unsuccessful, more invasive treatment are considered like transurethral resection of the prostate (TURP).

Both selective and less-selective $\alpha$-blockers may be associated with dizziness, asthenia, and postural hypotension, limiting therapy. Decreased libido and impotence are more common in men taking finasteride [17]. Although, transurethral resection is an effective treatment for symptomatic $\mathrm{BPH}$, approximately $15 \%$ to $25 \%$ of patients who undergo surgery do not have satisfactory longterm outcomes [18]. Because of these problems and the desire of patients to avoid surgery whenever possible, there has been much interest in alternative treatments. In the present study, a polyherbal formulation is evaluated for its efficacy and safety. Good agricultural and collection practice (GACP) was followed during the collection and manufacture of this Ayurvedic formulation. Botanical identification and desired quality were in accordance with the guidelines of Pharmacopoeial Standards of an Ayurvedic formulations and were carried out by a quailfied chemist approved by the Food and Drug Administration. The formulation was finalised by a team of Ayurvedic Scientists after scanning Ayurvedic and contemporary scientific literatures. This formulation has been approved by regulatory authorities in India as an Ayurvedic formulation and is available for clinical practice.

The principal herbs of this polyherbal formulation include extracts of Curculigo orchioides, Lactuca scariola, Asteracantha longifolia, Mucuna pruriens, Parmelia perlata, Argyreia speciosa, Tribulus terrestris and Leptadenia reticulat (Table 1). The stability of this formulation was tested by accelerated study (upto 6 months) and real time study (upto 3 years).

Table 1. Composition of polyherbal formulation: each capsules contains.

\begin{tabular}{ccc}
\hline Plant Name & Quantity \\
\hline Mushali (Curculigo orchioides) & $32 \mathrm{mg}$ \\
Vanya kahu (Lactuca scariola) & $8 \mathrm{mg}$ \\
Kokilaksha (Asteracantha longifolia) & $16 \mathrm{mg}$ \\
${\text { Extracts* }{ }^{*}:}^{\text {Kapikachchu (Mucuna pruriens) }}$ & $20 \mathrm{mg}$ \\
& Shaileyam (Parmelia perlata) & $32 \mathrm{mg}$ \\
& Vriddhadaru (Argyreia speciosa) & $64 \mathrm{mg}$ \\
& Gokshura (Tribulus terrestris) & $64 \mathrm{mg}$ \\
Jeevanti (Leptadenia reticulata) & $64 \mathrm{mg}$ \\
\hline
\end{tabular}

${ }^{*}$ Herbs in the above formula are cleaned and reduced to a coarse powder in a pulverizer individually and blended in the ratio mentioned above. This herbal blend is extracted with demineralized water, filtered and concentrated. The extract is then granulated and reduced to required mesh size, blended and filled into capsules. 


\section{Aim of the Study}

This study was planned to evaluate the clinical efficacy and safety of a polyherbal formulation in the management of benign prostatic hyperplasia.

\section{Materials and Methods}

\subsection{Study Design}

This was a single blind, randomized and placebo-controlled clinical study conducted at Bharathi Raja Multispeciality Hospital, Chennai, India, as per WHO operational guidance to support clinical trials of herbal product [19]. Patients were unaware of the treatment assigned to them. The study protocol, case report forms, regulatory clearance documents, product related information and informed consent form were submitted to the "Institutional Ethics Committee" and were approved by the same.

\subsection{Sample Size Calculation}

Sample size was calculated based on the Nomogram for standardized difference of 0.83 with $90 \%$ power to detect the difference with a two-tailed $p$ value of 0.05 [20]. Based on this calculation, the sample size is approximately 58: i.e., 29 participants required for each arm of the study. The total sample size for this study was rounded off to 60 patients.

\subsection{Randomization}

Sixty patients were randomized into two groups of 30 in each of placebo and drug group. Randomization was carried out by sequential opening of sealed opaque envelopes (prepared by Dr. PS Patki) containing the random group allocation previously generated using random table and opened in numerical order as the subject entered the study by the chief investigator (Dr. R. Jeyaraman). Patients were free to withdraw from the study, if they so desired, without assigning any reason. Effectiveness of single blinding was checked by the chief investigator by questioning the patients during the course of the clinical trial.

\subsection{Inclusion Criteria}

A total of 60 patients, aged between 30 and 65 years, who were diagnosed as suffering from $\mathrm{BPH}$ and who were willing to give informed consent were included in the study. The patients were categorized by the "AUA symptom score index" as either mild ( 0 - 7 points), moderate (8 - 19 points) or severe (20 - 35 points) cases of BPH.

\subsection{Exclusion Criteria}

Patients with diabetes mellitus, prostatic carcinoma, prostatitis, carcinoma bladder, neurogenic bladder, stricture urethra, vesical calculus and those patients who were not willing to give informed consent were excluded from the study. Similarly patients with raised prostate surface antigen $(\geq 3.9 \mathrm{ng} / \mathrm{ml})$ were excluded from the study.

\subsection{Study Procedure}

At the initial randomization visit, a detailed medical history, with special emphasis on history of urinary symptoms (hesitancy, straining, intermittency, frequency, nocturia, poor flow and sensation of incomplete voiding) was obtained from all the patients. All the patients underwent a thorough systemic examination and digital rectal examination (DRE). Routine blood analysis, urinalysis and serum levels of PSA were done for all the patients. In addition, abdominal pelvic ultrasonography was done at entry and after completing the study to estimate the approximate prostate weight and size and to measure the post-void residual (PVR) volume. The severity of the urinary parameters was evaluated using AUA symptom score. All the patients were randomized arbitrarily using random table into either polyherbal group $(n=30)$ or placebo $(n=30)$. Each patient received either a polyherbal formulation or placebo in a dose of 2 capsules, twice a day with meals for two months. The outcome of each group was assessed by comparing urinary hesitancy, intermittency, straining during micturition, urine flow, sense of incomplete micturition, frequency of nocturnal micturition, AUA symptom scores and PVR volume.

\subsection{Primary and Secondary Endpoints}

The predefined primary efficacy endpoints were, evaluation of AUA score, PVR urine, urinary flow rate and quality of life. The predefined secondary safety endpoints were evaluation of clinical and biochemical safety profile.

\subsection{Follow-Up}

Patients underwent the same evaluations after treatment as were performed at baseline, regardless of their treatment received and outcome. Compliance to drug therapy was evaluated by asking the patients to return back the investigational product container and counting the number of the remaining capsules.

\subsection{Adverse Events}

All adverse events, either reported or observed by patients, were recorded with information about severity, date of onset, duration, and action taken regarding the study drug. Relation of adverse events to the study medication was predefined as "Unrelated" (follows a reasonable temporal sequence from the administration of the 
drug), "Possible" (follows a known response pattern to the suspected drug, but could have been produced by the patient's clinical state or other modes of therapy administered to the patient), and "Probable" (follows a known response pattern to the suspected drug that could not be reasonably explained by the known characteristics of the patient's clinical state).

Patients were allowed to voluntarily withdraw from the study, if they so desired without assigning reasons. For patients withdrawing from the study, efforts were made to ascertain the reason for dropout. Non compliance (defined as failure to take less than $80 \%$ of the medication) was not regarded as treatment failure, and reasons for non compliance were noted.

\subsection{Statistical Analysis}

Statistical analysis was done according to the intentionto-treat principles using GraphPad Prism version 4.03, for windows (GraphPad software, San Diego, California USA). The analysis included all randomized subjects in the groups to which they were allocated and the missing responses was evaluated using the last observation carry forward method (LOCF). Changes in various parameters were assessed for statistical evaluation using between the group analysis at entry and at the end of the study. Fisher's exact test was used for urinary symptoms and unpaired " $\mathrm{t}$ " test (Independent t-test) for AUA symptom score, prostate weight and postvoid residual volume. Minimum level of significance was fixed at $95 \%$ confidence limit and two-sided $p<0.05$ was considered significant.

\section{Results}

Sixty consecutive patients were enrolled into the trial between December 2007 and March 2009 were randomly divided into two groups of 30 each. Mean age was 47.00 \pm 10.80 years in polyherbal group and $50.30 \pm 6.50$ years in placebo group. In polyherbal formulation group, 12 patients were smokers, 6 were alcoholics and 17 patients were vegetarians. In placebo group, 11 patients were smokers, 8 were alcoholics and 22 patients were vegetarians. There was no statistical difference between the polyherbal formulation and placebo groups (Table 2).

Data of the 56 patients was available for analysis as four patients from the placebo group withdrew from the study due to lack of benefit to the treatment (the missing responses were evaluated using the LOCF method). The baseline values (on entry) for various parameters in the placebo and polyherbal treatment groups were not significant. There was significant reduction in the presence of urinary hesitancy in the polyherbal treatment group (8/19 patients) as compared to placebo (14/17 patients) $(p<0.01)$ at the end of treatment and reduction in intermittent urinary flow in the polyherbal treatment group (7/16 patients) as compared to placebo (19/21 patients) $(p<0.003)$. Polyherbal treatment group showed significant improvement in parameters such as straining during urination ( $p<0.001)$, poor flow $(p<0.005)$ and sense of incomplete micturition $(\mathrm{p}<0.02)$ as compared to the placebo group (Table 3).

Table 2. Demographic data of patients on entry $(n=60)$.

\begin{tabular}{lcc}
\hline Parameters & $\begin{array}{c}\text { Polyherbal } \\
\text { formulation }\end{array}$ & Placebo \\
\hline No. of patients on entry & 30 & 30 \\
No. of patients withdrawn & 0 & 4 \\
Mean age (years) (Mean \pm SD) & $47.00 \pm 10.80$ & $50.30 \pm 6.50$ \\
Smokers (No. of patients) & 12 & 11 \\
Alcohol (No. of patients) & 6 & 8 \\
Diet (veg.) & 17 & 22 \\
\hline
\end{tabular}

Table 3. Effect of polyherbal formulation and placebo on urinary symptoms in patients with BPH.

\begin{tabular}{|c|c|c|c|c|c|c|}
\hline \multirow{2}{*}{ Symptoms } & \multicolumn{2}{|c|}{$\begin{array}{l}\text { No. of patients showing symptoms } \\
\text { on entry }\end{array}$} & \multirow{2}{*}{ p value } & \multicolumn{2}{|c|}{$\begin{array}{c}\text { No. of patients showing symptoms at the } \\
\text { end of treatment }\end{array}$} & \multirow{2}{*}{ p value } \\
\hline & Polyherbal formulation & Placebo & & Polyherbal formulation & Placebo & \\
\hline Hesitancy of urination & 19 & 17 & NS & 08 & 14 & 0.01 \\
\hline Intermittent flow & 16 & 21 & NS & 07 & 19 & 0.003 \\
\hline Straining during urination & 26 & 22 & NS & 19 & 22 & 0.0001 \\
\hline Poor flow & 17 & 15 & NS & 06 & 13 & 0.005 \\
\hline Sense of incomplete micturition & 24 & 20 & NS & 13 & 18 & 0.02 \\
\hline Nocturia & 23 & 25 & NS & 14 & 22 & 0.013 \\
\hline
\end{tabular}

NS: Not significant; Statistical analysis: Fisher's exact test. 
In polyherbal formulation group, nocturia (frequency of night-time urination) was significantly reduced in comparison to placebo group (Table 3, $\mathrm{p}<0.013$ ).

AUA symptom score and PVR volume showed significant reductions in the polyherbal treatment group as compared to placebo at the end of treatment $(p<0.0001)$. Also prostate weight was reduced after treatment with polyherbal formulation as compared to placebo, though it was not statistically significant (Table 4).

Assessment of questionnaires revealed an improvement in the QOL observed in Prostacare group as compared to placebo group. This was further supported by the findings which included AUA score, frequency of urination and nocturia (Tables 3 and $\mathbf{4}$ ).

No adverse effects were reported with polyherbal formulation during the entire study period except slight to notable hot flushes reported by 2 patients during polyherbal formulation therapy, which subsided at the end of the study period and did not necessitate the withdrawal of the polyherbal formulation.

\section{Discussion}

Benign prostatic hyperplasia refers to an anatomic diagnosis, in practice it is typically diagnosed clinically on the basis of lower urinary tract symptoms and prostatic enlargement detected on manual rectal examination or transrectal ultrasonograph [21]. Blocking androgen receptor activity is a common therapeutic course for $\mathrm{BPH}$ [22]. This approach however, results in several side effects, and most notably erectile dysfunction [23,24]. The risks and complications associated with TURP include post-operative hemorrhage, TUR-syndrome, urinary tract infection, retrograde ejaculation, bladder neck contractures and urethral strictures.

In the present study, polyherbal formulation was evaluated for the beneficial actions on the prostate. Curculigo orchioides is effective mainly on the urinary system and it is given in dysuria, and in poluria. It has $5 \alpha$-reductase inhibitor activity that helps to manage prostatic enlargement [25]. Lactuca scariola exhibits antibacterial activity that may be helpful in preventing the chronic urinary infection as seen in BPH [26]. As- teracantha longifolia has antimicrobial activity that may be helpful in infections caused due to stasis of urination along with infection in the hyperplasic prostatic mass in BPH [27].

Mucuna pruriens roots are used for diseases of renal and prostatic affections [28]. It also possesses anti-inflammatory activity that may be helpful in providing symptomatic relief in $\mathrm{BPH}$ [29]. On administration of Mucuna pruriens extract improvement in serum creatinine and urea, showing potent nephroprotective activity have been observed [30]. Parmellia perlata is active against common pathogens [31]. It contain acidic substance that has been used as an antimicrobial agent especially against Salmonella aureus that is implicated in mixed infections and has shown high level of resistance to the commonly marketed antibiotics. This activity stands may be helpful in providing symptomatic relief from symptoms of infections in prostatic mass, urinary bladder, and kidney due to susceptible organism [32].

Argyreia speciosa root has diuretic and aphrodisiac activities. Also it is effective in infection caused by common pathogens due to its antimicrobial activity and may be beneficial in management of BPH [33]. A. speciosa possesses anti-inflammatory activity and support the rationale behind the traditional use of these plants in inflammatory conditions and may be useful in BPH [34].

Tribulus terrestris exerts strong anti-inflammatory activity, and provide beneficial effect in BPH [35]. The fruits are regarded as diuretic, tonic, and are used in painful micturition, calculus affections, and urinary disorders [36]. Serum luteinizing hormone may play a role in control of luteinizing hormone (LH) as in some investigation, it is found with low LH in cases of BPH [37]. Tribulus terrestris shows potential increase in $\mathrm{LH}$ and may play role in controlling prostatic hyperplasia in $\mathrm{BPH}$ [38]. Leptadenia reticulata herb is used as stimulant and restorative. It is also used in dysuria, which may be helpful in BPH $[39,40]$. This herb has bacteriocidal activity and used in inflammatory conditions of prostate [41].

Herbal formulations like Saw Palmetto blend have been used to evaluate their safety and efficacy [42]. An

Table 4. Effect of polyherbal formulation and placebo on AUA symptom score, ultrasonography and uroflowmetry parameters.

\begin{tabular}{|c|c|c|c|c|c|c|}
\hline \multirow{2}{*}{ Parameters } & \multicolumn{2}{|l|}{ On entry } & \multirow{2}{*}{ p value } & \multicolumn{2}{|c|}{ At the end of treatment } & \multirow{2}{*}{ p value } \\
\hline & Polyherbal formulation & Placebo & & Polyherbal formulation & Placebo & \\
\hline AUA symptom score & $14.60 \pm 3.20$ & $13.80 \pm 4.60$ & NS & $9.2 \pm 2.60$ & $12.90 \pm 3.80$ & 0.0001 \\
\hline Prostate weight (gms) & $34.60 \pm 8.70$ & $32.60 \pm 8.30$ & NS & $32.60 \pm 9.40$ & $34.50 \pm 6.60$ & NS \\
\hline Post-void residual (PVR) volume (ml) & $85.30 \pm 20.20$ & $86.20 \pm 18.20$ & NS & $52.10 \pm 23.10$ & $89.70 \pm 28.30$ & 0.0001 \\
\hline
\end{tabular}

NS: Not significant; Values are indicated in Mean $\pm \mathrm{SD}$; Statistical analysis: unpaired " $\mathrm{t}$ " test (independent $\mathrm{t}$-test). 
analysis has also been carried out to evaluate costs of alternate treatments for benign prostate hypertrophy [43]. Herbal therapy has been a favored therapy for their safety.

The beneficial actions observed in this study in the management of BPH could be due to the synergistic actions of the potent herbs of polyherbal formulation which include $5 \alpha$-reductase inhibitor activity of Curculigo orchioides, bacteriocidal and antimicrobial activity of Lactuca scariola, Asteracantha longifolia, Argyreia speciosa and Parmellia perlata, anti-inflammatory activity of Mucuna prurien, Argyreia speciosa, Tribulus terrestris and Leptadenia reticulata, LH enhancing activity of Tribulus terrestris and nephroprotective activity of $\mathrm{Mu}$ cuna pruriens.

The study outcome was found to be beneficial in the management of moderate $\mathrm{BPH}$, however, it has some limitations, like a need for larger multicentric trials to further confirm these findings.

\section{Conclusion}

This study was planned to evaluate the clinical efficacy and safety of polyherbal formulation in BPH. Present study observed a significant reduction in the mean AUA symptom score, PVR volume and significant reduction in urinary hesitancy, intermittent flow, straining during urination, poor flow, sense of incomplete micturition and frequency of night-time urination, in the polyherbal formulation group, which indicate clinically beneficial effects of polyherbal formulation in BPH. Polyherbal formulation was well tolerated and safe. The beneficial clinical efficacy of polyherbal formulation in the management of BPH could be due to the synergistic actions of its potent herbs. Therefore, it may be concluded that use of polyherbal formulation is clinically effective and safe in the management of BPH. However, a larger study is needed to confirm these findings. We also warn that raised PSA levels need to be ruled out before starting this drug therapy.

\section{Acknowledgements}

Authors thank Dr. Rangesh Paramesh. MD (Ayur) for his help in this study.

\section{REFERENCES}

[1] H. M. Arrighi, E. J. Metter, H. A. Guess and J. L. Fozzard, "Natural History of Benign Prostatic Hyperplasia and Risk of Prostatectomy: The Baltimore Longitudinal Study of Aging," Urology, Vol. 38, No. 1, 1991, pp. 4-8. doi:10.1016/0090-4295(91)80191-9

[2] S. J. Berry, D. S. Coffey, P. C. Walsh and L. L. Ewing, "The Development of Human Benign Prostatic Hyperplasia with Age," Journal of Urology, Vol. 132, 1984, pp.
474-479.

[3] J. E. Oesterling, "Benign Prostatic Hyperplasia-Medical and Minimally Invasive Treatment Options," The New England Journal of Medicine, Vol. 332, 1995, pp. 99-110. doi:10.1056/NEJM199501123320207

[4] J. D. McConnell, "The Pathophysiology of Benign Prostatic Hyperplasia," Journal of Anthology, Vol. 12, 1991, pp. 356-363.

[5] M. H. Minsley and S. Wrenn, "Long-Term Care of the Tracheostomy Patient from an Outpatient Perspective," ORL_Head and Neck Nursing, Vol. 14, No. 4, 1996, pp. 18-22.

[6] N. K. Roberts, "The Selective Approach to Successful Stomal Management at Home," ORL-Head and Neck Nursing, Vol. 13, No. 4, 1995, pp. 12-16.

[7] T. J. Beckman and L. A. Mynderse, "Evaluation and Medical Management of Benign Prostatic Hyperplasia," Mayo Clinic Proceedings, Vol. 80, No. 10, 2005, pp. 1356-1362. doi: $10.4065 / 80.10 .1356$

[8] M. T. Rosenberg, D. R. Staskin, S. A. Kaplan, S. A. MacDiarmid, D. K. Newman and D. A. Ohl, "A Practical Guide to the Evaluation and Treatment of Male Lower Urinary Tract Symptoms in the Primary Care Setting," International Journal of Clinical Practice, Vol. 61, 2007, pp. 1535-1546. doi:10.1111/j.1742-1241.2007.01491.x

[9] AUA Practice Guidelines Committee, "AUA Guideline on Management of Benign Prostatic Hyperplasia: Diagnosis and Treatment Recommendations," Chapter 1, 2003, pp. 1-11. http://professional.medtronic.com

[10] C. G. Roehrborn, "The Potential of Serum Prostate-Specific Antigen as a Predictor of Clinical Response in Patients with Lower Urinary Tract Symptoms and Benign Prostatic Hyperplasia," British Journal of Urology International, Vol. 93, 2004, pp. 21-26.

[11] J. A. Schalken, "Molecular and Cellular Prostate Biology: Origin of Prostate-Specific Antigen Expression and Implications for Benign Prostatic Hyperplasia," British Journal of Urology International, Vol. 93, 2004, pp. 5-9. doi:10.1111/j.1464-410X.2003.04633.x

[12] M. J. Barry and C. G. Roehrborn, "Benign Prostatic Hyperplasia," British Medical Journal, Vol. 323, 2001, pp. 1042-1046. doi:10.1136/bmj.323.7320.1042

[13] C. R. Chapple, "Pharmacological Therapy of Benign Prostatic Hyperplasia/Lower Urinary Tract Symptoms: An Overview for the Practising Clinician," British Journal of Urology International, Vol. 94, 2004, pp. 738-744. doi:10.1111/j.1464-410X.2004.05022.x

[14] C. L. Eaton, "Aetiology and Pathogenesis of Benign Prostatic Hyperplasia," Current Opinion in Urology, Vol. 13, 2003, pp. 7-10. doi:10.1097/00042307-200301000-00002

[15] T. J. Beckman and L. A. Mynderse, "Evaluation and Medical Management of Benign Prostatic Hyperplasia," Mayo Clinic Proceedings, Vol. 80, No. 10, 2005, pp. 13561362. doi: $10.4065 / 80.10 .1356$

[16] A. Tubaro and C. D. Nunzio, "The Current Role of Open Surgery in BPH," EAU-EBU Update Series, Vol. 4, 2006, pp. 191-201. doi:10.1016/j.eeus.2006.07.002

[17] G. Maria, G. Brisinda, I. M. Civello, A. R. Bentivoglio, G. 
Sganga and A. Albanese, "Relief by Botulinum Toxin of Voiding Dysfunction Due to Benign Prostatic Hyperplasia: Results of a Randomized, Placebo-Controlled Study Giorgio," Urology, Vol. 62, No. 2, 2003, pp. 259-265. doi:10.1016/S0090-4295(03)00477-1

[18] G. L. Lu-Yao, M. J. Barry, C. H. Chang, et al., "For the Prostate Patient Outcomes Research Team (PORT): Transurethral Resection of the Prostate among Medicare Beneficiaries in the United States: Time Trends and Outcomes," Urology, Vol. 44, 1994, pp. 692-698. doi:10.1016/S0090-4295(94)80208-4

[19] Operational Guidance, "Information Needed to Support Clinical Trials of Herbal Products," World Health Organization on Behalf of the Special Programme for Research and Training in Tropical Diseases, 2005, TDR/GEN/Guidance/05.1.

[20] E. Whitley and J. Ball, "Statistics Review 4: Sample Size Calculations," Critical Care, Vol. 6, 2002, pp. 335-341. doi:10.1186/cc1521

[21] J. D. McConnell, M. J. Barry and R. C. Bruskewitz, "Benign Prostatic Hyperplasia: Diagnosis and Treatment (Clinical Practice Guideline No. 8)," In: M. D. Rockville, Ed., Agency for Health Care Policy and Research, Public Health Service, US Department of Health and Human Services, 1994.

[22] J. D. McConnell, "Androgen Ablation and Blockade in the Treatment of Benign Prostatic Hyperplasia," Urologic Clinics of North America, Vol. 17, 1990, pp. 661-670.

[23] E. Stoner, "The Clinical Development of a 5 Alpha-Reductase Inhibitor, Finasteride," The Journal of Steroid Biochemistry and Molecular Biology, Vol. 37, 1990, pp. 375-378. doi:10.1016/0960-0760(90)90487-6

[24] G. Bartsch, R. S. Rittmaster and H. Klocker, "Dihydrotestosterone and the Concept of 5Alpha-Reductase Inhibition in Human Benign Prostatic Hyperplasia," World Journal of Urology, Vol. 19, 2002, pp. 413-425.

[25] R. N. Biswas and S. O. Temburnikar, "Safed Musali (Chlorophytum Species)-A Wonder Drug in the Tropical Zone," XIth World Foresty Conference, Canada, 2003.

[26] R. N. Yadava and J. Jharbade, "New Antibacterial Triterpenoid Saponin from Lactuca scariola," Fitoterapia, Vol. 79, No. 4, 2008, pp. 245-249. doi:10.1016/i.fitote.2007.11.028

[27] R. P. Samy, "Antimicrobial Activity of Some Medicinal Plants from India," Fitoterapia, Vol. 76, No. 7-8, 2005, pp. 697-699. doi:10.1016/j.fitote.2005.06.011

[28] S. N. Yoganarasimhan, "Mucuna pruriens. Medicinal Plants of India. Tamil Nadu," Bangalore, Vol. 2, 2000, p. 366.

[29] V. N. Pandey, "Pharmacological investigations of Certain Medicinal Plants \& Compound Formulations Used in Ayurveda \& Siddha," CCRAS, Department of ISM \& H. Government of India, New Delhi, 1996, pp. 69-72.

[30] G. K. A. Adepoju and O. O. Oduben, "Effect of Mucuna pruriens on Some Hematological and Biochemical Pa- rameters," Journal of Medicinal Plants Research, Vol. 3, No. 2, 2009, pp. 73-76.

[31] C. P. Khare, "Parmelia perlata. Encyclopedia of Indian Medicinal Plants," Springer, Berlin, 2004, pp. 352-353.

[32] M. A. Momoh and M. U. Adikwu, "Evaluation of the Effect of Colloidal Silver on the Antibacterial Activity of Ethanolic Extract of Lichen Parmelia perlata," African Journal of Pharmacy Pharmacology, Vol. 2, No. 6, 2008, pp. 106-109.

[33] L. V. Asolkar, K. K. Kakkar and O. J. Chakre, “Argyreia speciosa. Second Supplement to Glossary of Indian Medicinal Plants with Active Principles," CSIR, Government of India, New Delhi, 1992, p. 87.

[34] A. B. Gokhale, A. S. Damre, K. R. Kulkami and M. N. Saraf, "Preliminary Evaluation of Anti-Inflammatory and Anti-Arthritic Activity of S. lappa, A. speciosa and $A$. aspera," Phytomedicine, Vol. 9, No. 5, 2002, pp. 433-437. doi:10.1078/09447110260571689

[35] C. H. Hong, S. K. Hur, O. J. Oh, S. S. Kim, K. A. Nam and S. K. Lee, "Evaluation of Natural Products on Inhibition of Inducible Cyclooxygenase (COX-2) and Nitric Oxide Synthase (iNOS) in Cultured Mouse Macrophage Cells," Journal of Ethnopharmacology, Vol. 83, No. 1-2, 2002, pp. 153-159. doi:10.1016/S0378-8741(02)00205-2

[36] R. N. Chopra, I. C. Chopra, K. L. Handa and L. D. Kapur, "Tribulus terrestris. Chopra's Indigenous Drugs of India," U N Dhur \& Sons Private Limited, Kolkata, 1958, p. 430.

[37] G. L. Hammond, O. Lukkarinen, P. Vihko, M. Kontturi and R. Vihko, "The Hormonal Status of Patients with Benign Prostatis Hypertrophy: FSH, LH, TSH and Prolactin Responses to Releasing Hormones," Clinical Endocrinology, Vol. 10, No. 6, 1979, pp. 545-552.

[38] M. L. Kuhut, et al., "Ingestion of a Dietary Supplement Containing Dehydroepiandrosterone (DHEA) and Androstenedione Has Minimal Effect on Immune Function in Middle-Aged Men," Journal of the American College of Nutrition, Vol. 22, No. 5, 2003, pp. 363-371.

[39] C. P. Khare, "Leptadenia reticulata. Indian Medicinal Plants: Illustrated Dictionary," Springer, Berlin, 2007, p. 371.

[40] S. N. Yoganarasimhan, "Leptadenia reticulata. Medicinal Plants," Vol. 2, Tamil Nadu, Bangalore, 2000, p. 322.

[41] F. M. Parabia, et al., "Effect of Plant Growth Regulators on in Vitro Morphogenesis of Leptadenia reticulata (Retz.) W. and A. from Nodal Explants," Current Sciences, Vol. 92, No. 9, 2007, pp. 1290-1293.

[42] L. S. Marks, et al., "Effects of a Saw Palmetto Herbal Blend in Men with Symptomatic Benign Prostatic Hyperplasia," Journal of Urology, Vol. 163, 2000, pp. 14511456. doi:10.1016/S0022-5347(05)67641-0

[43] J. A. Vale, et al., "An Analysis of the Costs of Alternative Treatments for Benign Prostatic Hypertrophy," Journal of the Royal Society of Medicine, Vol. 88, No. 11, 1995, pp. 644-648. 